\title{
Novel Asthma Therapeutics: Insights from Whole-Genome Studies
}

\author{
Ting F Leung*, Man F Tang, Hing Y Sy and Gary WK Wong
}

Department of Pediatrics, The Chinese University of Hong Kong, Prince of Wales Hospital, Hong Kong SAR, People's Republic of China

\begin{abstract}
Asthma is characterized by recurrent and reversible airflow obstruction as well as bronchial hyper-responsiveness. Airway inflammation is central to asthma pathogenesis. Bronchodilators are advocated as rescue treatments for acute asthma symptoms, whereas anti-inflammatory drugs such as Inhaled Corticosteroids (ICS) are common controller therapies for chronic asthma. Leukotriene modifiers are widely prescribed alternatives to ICS. During the past several years, a number of pharmacogenomic studies adopting whole-genome arrays have identified novel gene targets that contribute to the heterogeneity in responses to these anti-asthma drugs. These gene chips contain dense probes that capture either genotypes of single-nucleotide polymorphisms or the expression of genes across the entire human genome. Through these approaches, CLCA1, periostin, serpinB2, FKBP51, NFKB, GLCCI1 and T gene were reported to modulate ICS response in asthma patients whereas ARG1, CRHR2, SPATS2L and COL22A1were novel genes for bronchodilator responses. Some of these therapeutic targets were replicated in independent populations and/or supported by downstream in vitro and in vivo experiments on their functionality. Adequate bioinformatics support was essential in pharmacogenomics research in view of the enormous amount of whole-genome data involved. These whole-genome findings will ultimately facilitate personalized asthma pharmacotherapy that allows us to choose among treatment options that will likely be effective to any particular patient. However, more resources and collaborative efforts are required to advance the pharmacogenomics research.
\end{abstract}

Keywords: Asthma; Bronchodilator; Inhaled corticosteroid; Genomics; Leukotriene modifier; Pharmacogenetics; Therapeutic response

\section{Introduction}

Asthma is a very prevalent respiratory disease affecting more than 300 million people worldwide [1]. These patients suffer from recurrent cough, dyspnea and wheeze caused by reversible airflow limitation, which is due to Bronchial Hyper Responsiveness (BHR) of their airways to allergens and other environmental stimuli. Asthma is the result of complex interactions between many genetic and environmental factors [2-4]. Airway inflammation is a central feature of asthma pathogenesis, which is readily detectable by a variety of monitoring methods ranging from non-invasive exhaled breath analyses through induced sputum to invasive bronchial biopsy and broncho alveolar lavage. Anti-inflammatory therapies are therefore an integral component of asthma pharmacotherapy under different international guidelines $[5,6]$, under which asthmatic patients are treated with a combination of different short-term and long-term medications including inhaled corticosteroids (ICS), bronchodilators and leukotriene modifiers. Whereas these treatments have proven efficacy for asthma as a whole, there is substantial inter-individual variability in patients' response to each of the above three major classes of asthma drugs $[7,8]$.

Pharmacogenetics is the study of genetic determinants for the heterogeneity of responses to asthma medications by the analysis of a gene-environment interaction where the environment is exposed to a pharmacological therapy. This investigative approach greatly facilitates personalized medicine by differentiating treatment responders from non-responders, whereby substantially reducing the economic burden of asthma. On the other hand, such benefits of pharmacogenetic testing have to be balanced against the genotyping cost, willingness of testing and availability of genomic data for trait mapping for individual patients. Candidate gene and pathway-based pharmacogenetic approaches have already identified a number of candidate genes and their functional variants, but larger-scale pharmacogenomics has drawn more and more attention over the past decade due to the decreasing cost of dense arrays with hundreds of thousands of Single-Nucleotide Polymorphisms
(SNPs) and rapid advances in high-throughput genotyping technology. The ultimate aim of these pharmacogenomics studies is to formulate a better prognostic model for therapeutic response in asthma. Herein, this article reviewed recent evidence on pharmacogenomics of ICS, bronchodilators and leukotriene modifiers that brings us closer to an era of personalized asthma therapy. Table 1 summarizes the findings of whole-genome pharmacogenomic studies for asthma and comparison of minor allele frequencies of the novel SNPs among different ethnic groups according to the 1000 Genomes Project [9].

\section{Inhaled Corticosteroids}

ICSs are considered as the most effective and commonly used anti-inflammatory drugs for asthma [10]. The combination of high inter-individual variability $[11,12]$ and low intra-individual variability [7] among patients who received ICS supports a genetic basis for the treatment response to ICS. Asthma patients who are less responsive to ICS can be advised to take other forms of controller therapies [5]. Woodruff et al. found significant association by genome-wide profiling between ICS response and the expression of epithelial cell genes [13]. They collected bronchial epithelial cells from 42 asthmatics, 28 non-smoking healthy subjects and 16 smokers without asthma. By examining expression profiles on genome-wide microarrays, they demonstrated that ICS treatment down-regulated expression of chloride channel, calcium-activated, family member 1 (CLCA1), periostin, and serine peptidase inhibitor, clade B (ovalbumin), member

${ }^{*}$ Corresponding author: Ting Fan Leung, Department of Pediatrics, 6/F, LuiChe Woo Clinical Sciences Building, Prince of Wales Hospital, Shatin, Hong Kong SAR, People's Republic of China, Tel: +(852) 2632 2981; Fax: +(852) 2636 0020; E-mail: tfleung@cuhk.edu.hk

Received April 09, 2013; Accepted May 04, 2013; Published May 10, 2013

Citation: Leung TF, Tang MF, Sy HY, Wong GWK (2013) Novel Asthma Therapeutics: Insights from Whole-Genome Studies. J Pharmacogenom Pharmacoproteomics 4: 115. doi:10.4172/2153-0645.1000115

Copyright: $\odot 2013$ Leung TF, et al. This is an open-access article distributed under the terms of the Creative Commons Attribution License, which permits unrestricted use, distribution, and reproduction in any medium, provided the original author and source are credited. 


\begin{tabular}{|c|c|c|c|c|c|c|c|c|c|}
\hline \multirow[t]{2}{*}{ Locus [ref] } & \multirow[t]{2}{*}{ SNP } & \multirow[t]{2}{*}{ Gene } & \multirow{2}{*}{$\begin{array}{c}\text { rs } \\
\text { number }\end{array}$} & \multirow{2}{*}{$\begin{array}{l}\text { Effect for increased expression or minor } \\
\text { allele }\end{array}$} & \multirow[t]{2}{*}{ Minor allele } & \multicolumn{4}{|c|}{$\begin{array}{l}\text { Published MAFs in different ethnic groups } \\
\text { according to } 1000 \text { Genomes Project [9] }\end{array}$} \\
\hline & & & & & & $\mathrm{CHB}$ & JPT & CEU & YRI \\
\hline Chr 1 [13] & NA & CLCA1* & NA & Good ICS response & NA & NA & NA & NA & NA \\
\hline Chr 13 [13] & NA & Periostin* & NA & Good ICS response & NA & NA & NA & NA & NA \\
\hline Chr 18[13] & NA & SerpinB2* & NA & Good ICS response & NA & NA & NA & NA & NA \\
\hline Chr6[13] & NA & FKBP51* & NA & Poor ICS response & NA & NA & NA & NA & NA \\
\hline Chr 4[14] & NA & $N F K B^{*}$ & NA & Poor ICS response & NA & NA & NA & NA & NA \\
\hline Chr 7 [15] & A-549G & GLCCI1 ${ }^{\dagger}$ & 37973 & Increased ICS response & G & 0.464 & 0.421 & 0.482 & 0.159 \\
\hline Chr 6 [17] & $\mathrm{G}+11024 \mathrm{~T}$ & T-gene $^{\dagger}$ & 1134481 & Decreased ICS response & $\mathrm{T}$ & 0.160 & 0.208 & 0.347 & 0.227 \\
\hline Chr $6[27,32]$ & A-2464G & $A R G 1^{\dagger}$ & 2781659 & Asthma susceptibility and increased BDR & G & 0.289 & 0.365 & 0.282 & 0.670 \\
\hline Chr 2 [34] & C-20564T & SPATS $2 L^{\dagger}$ & 295137 & Increased $\beta_{2}$-adrenoceptor levels and BDR & A & 0.191 & 0.225 & 0.429 & 0.574 \\
\hline Chr 8 [18] & $\mathrm{C}+41740 \mathrm{~T}$ & COL22A $1^{\dagger}$ & 6988229 & IncreasedBDR & $\mathrm{T}$ & 0 & 0.011 & 0.247 & 0.608 \\
\hline
\end{tabular}

Table 1: Summary of major findings from whole-genome pharmacogenomic studies for asthma

A, adenine; BDR, bronchodilator responsiveness; CEU, nnorthern and western European ancestry (CEU); CHB, Han Chinese in Beijing, China; Chr, chromosome; G, guanine; ICS, inhaled corticosteroid; JPT, Japanese in Tokyo, Japan; MAF, minor allele frequency; NA, not applicable; T, thymine; UTR, untranslated region; YRI, Yoruba in Ibadan, Nigeria.

*By whole-genome microarray; ${ }^{\star}$ By genome-wide association study.

2 (serpinB2) while up-regulated expression of FK506-binding protein 51 (FKBP51) in asthmatic subjects. High baseline expression of CLCA1, periostin and $\operatorname{serpin} B 2$ was correlated with a good clinical response to ICS, whereas high expression of FKBP51 was associated with a poor response. Another study exploring genome-wide gene expression profiles in Peripheral Blood Mononuclear Cells (PBMC) derived a prediction model of glucocorticoid (GC) sensitivity in asthma patients [14]. In this study, the expression of 11,812 genes was examined with high-density oligonucleotide microarrays in two independent cohorts of 64 GC-sensitive and 42 GC-resistant asthma patients. Expression of 11 differentially expressed genes could be used to predict GC responders and non-responders among asthma patients with $84 \%$ accuracy. Among these genes, the one encoding DNA binding subunit of NF- $\mathrm{KB}(N F K B)$ was the best genetic predictor for GC responsiveness. This gene alone had $81 \%$ accuracy in differentiating GC-sensitive from GC-resistant patients.

In recent years, Genome-Wide Association Studies (GWAS) have gained increasing attention in dissecting the genetic basis of complex disease traits. Tantisira and coworkers genotyped 534,290 SNPs in 422 parent-child trios who participated in the Childhood Asthma Management Program (CAMP) [15].The associations between ICS response and top SNPs identified in initial GWAS were replicated in four independent populations totaling 935 persons. A functional SNP (rs37973) of glucocorticoid-induced transcript 1 gene (GLCCI1) was shown to be associated with substantial decrements in response to ICS treatment in asthma patients. Those homozygous for the minor allele of this SNP had higher ICS response than patients who were homozygous for the wild-type allele. The importance of GLCCI1rs37973 was examined in dataset of four clinical trials of asthmatics who received ICS [16]. This SNP and rs 1876828 of the gene encoding corticotrophin releasing hormone receptor 1 could be combined to yield $66 \%$ predictive performance of forced expiratory volume in 1-second $\left(\mathrm{FEV}_{1}\right)$ change following ICS treatment. The same group of researchers reported recently another pharmacogenomic study in which a transcription factor known as T gene was discovered to be a novel locus for ICS response in asthmatic subjects [17]. A total of 444,088 SNPs were genotyped in 418 white asthmatics. The top 50 SNPs were then tested in an independent clinical trial population of 407 white patients with asthma whose mean age was 39.4 years. They received 8 -week treatment with ICS, and their mean baseline $\mathrm{FEV}_{1}$ was $71.7 \%$. Combining the data from two populations, they found two variant rs3127412 and rs6456042to show the strongest association with patients' ICS response. Although these two SNPs were not located within a gene, they were tightly correlated with three variants in potentially functional regions within the $\mathrm{T}$ gene. After genotyping, each of these $\mathrm{T}$ gene variants was also associated with ICS response. A two-fold to three-fold difference in $\mathrm{FEV}_{1}$ response was noted between subjects homozygous for the wild-type and minor alleles. Such findings also strongly supported the usefulness of GWAS in identifying novel pharmacogenetic targets in asthma.

\section{Bronchodilators}

Short- and fast-acting bronchodilators are advocated as rescue treatment across the whole severity spectrum of asthma according to international guidelines [5,6]. They are the most widely prescribed anti-asthma drugs, which acts by relaxing airway smooth muscle and reversing airflow limitation. $\beta_{2}$-agonists are the most commonly used bronchodilators for treating asthma [18], which can be divided into two classes: the Short-Acting $\beta_{2}$-Agonists (SABAs) such as albuterol, fenoterol, pirbuterol, isoproterenol and levalbuterol as well as the LongActing $\beta_{2}$-Agonists (LABAs) such as salmeterol and formoterol. SABAs are the mainstay of treatment for rescuing acute asthma symptoms caused by bronchospasm whereas LABAs are mostly prescribed as controller anti-asthma treatment in combination with ICS in view of their superior response on asthma outcomes when compared to cysteinyl leukotriene 1 receptor antagonists (LTRAs) add-on [8]. LABA monotherapy in chronic asthma is not advocated because of reports of adverse outcomes associated with such treatment approach $[19,20]$.

Earlier pharmacogenetic studies focused on SNPs located in the gene encoding $\beta_{2}$-adrenoceptor (ADRB2) that mediates the pharmacological actions of $\beta_{2}$-agonists [21]. The Human Genome Epidemiology meta-analysis revealed two functional SNPs of ADRB2 (rs1042713 and rs1042714), corresponding to amino acids at positions 16 and 27 of $\beta_{2}$-adrenoceptor, to be important pharmacogenetic targets for both childhood and adult asthma [22]. Nonetheless, Taylor et al. could not observe any significant difference in bronchodilator response (BDR) with respect to any of the major $A D R B 2$ haplotypes or genotypes [23]. Analyses of findings from a double-blind study of 2,250 asthmatics and another open-label study of 405 asthmatics did not reveal any pharmacogenetic effect of $A D R B 2$ variation on therapeutic responses to salmeterol and formoterol [24]. A large clinical trial that randomized asthma patients according to their $A D R B 2$ genotypes also failed to detect different airway function in relation to ADRB2genotypes following ICS-LABA treatment [25]. In two large independent pharmacogenetic studies, Bleecker et al. failed to 
show significant effects of ADRB2 Gly16Arg genotype on therapeutic response and tolerability to long-term treatment with for moterol alone or in combination with budesonide among patients with moderate to very severe chronic obstructive pulmonary disease [26]. Because of these findings, recent pharmacogenomic studies have been trying to identify other genes that influence BDR of asthma patients.

Litonjua et al. explored family-based association testing between BDR to $\beta_{2}$-agonists and SNPs of arginase 1 gene (ARG1) [27]. They adopted a novel screening algorithm that was implemented according to the statistical power of each SNP to rank 844 SNPs in111 candidate genes for BDR in 209 children and their parents who participated in the CAMP. Genes that had SNPs with median power in the highest quartile were subsequently selected for replication analyses in three other asthma cohorts. By combining evidence for association from all populations, these authors identified four SNPs from ARG1 (rs2781659, rs2781663, rs2781665, and rs2749935) to have the strongest associations with BDR. That of rs2781659 survived the stringent Bonferroni correction for multiple statistical testing. Three of the four SNPs (rs2781659, rs2781663, and rs2781665) were in strong linkage disequilibrium with each other.

This finding regarding the importance of $A R G 1$ was supported by earlier in vivo studies of murine asthma models in which increased expression of genes coding for two arginase isoforms was detected in lung tissue [28]. ARG1 expression was increased in bronchoalveolar lavage cells from asthmatic humans, and $A R G 1$ mRNA was strongly detected in human asthmatic lung biopsy tissue. Functionally, L-arginine significantly reduced BHR and airway inflammation in a murine model of asthma [29]. L-arginine reduced activity and expression of arginase- 1 and increased expression of endothelial nitric oxide synthase in bronchial epithelium, which was associated with increased exhaled nitric oxide levels and cyclic guanosine monophosphate in the lung and reduced biomarkers of nitro-oxidative stress. Another study also found $A R G 1$ to be over-expressed in human asthma whereas the expression of all other genes involved in L-arginine metabolism remained unchanged [30]. Breton et al. studied epigenetic variations of $A R G 1, A R G 2$ and nitric oxide synthase genes in 940 participants of the Children's Health Study [31]. They found the degree of DNA methylation in ARG2 to be associated with exhaled NO levels, and such association was larger in children with asthma than in non-asthmatic children. Exhaled NO levels of asthmatic children were influenced by DNA methylation in ARG1.

Our results also support $A R G 1$ to be an important candidate gene for asthma in southern Chinese, and add to the literature that $A R G 1$ interacts with the gene encoding corticotropin-releasing hormone receptor-2 (CRHR2) to modulate BDR in adults with asthma [32]. In this study, 15 SNPs from eight candidate genes for asthma and BDR were genotyped in 345 unrelated Chinese adults with physiciandiagnosed asthma and 464 non-allergic controls. Patients' mean FEV and $\mathrm{FEV}_{1} / \mathrm{FVC}$ were $88.6 \%$ predicted and 0.81 respectively. One quarter of them showed significant BDR to inhaled salbutamol. Regarding antiasthma treatment, $83 \%$ and $54 \%$ of these patients received ICS and LABA respectively, and $1.2 \%$ were treated with oral corticosteroid. All subjects were either non-smokers or ex-smokers for at least one year with a smoking history of less than 10 pack years. We found that asthma diagnosis was associated with rs7216389 of ORMDL3, the first asthma gene identified by GWAS [33], and rs3756780 of ARG1. BDR was associated with rs2749935 of ARG1.Generalized multifactor dimensionality reduction (GMDR) analyses for gene-gene interactions revealed that rs7216389 of ORMDL3 and rs3756780 of ARG1 might interact to determine asthma susceptibility. Subjects with high-risk genotypes from these two SNPs as assigned by GMDR method had an odds ratio 1.66 for asthma when compared with those with low-risk genotypes. Rs2749935 in ARG1 and rs2190242 in CRHR2 interacted to modulate BDR, and asthmatic patients with high-risk genotypes had larger $\mathrm{FEV}_{1}$ reversibility than low-risk patients and high-risk controls (mean $\mathrm{FEV}_{1}: 10.7 \%$ vs $6.8 \%$ vs $2.8 \%$ ).

A recent whole-genome pharmacogenomic study investigated genetic determinants of BDR using SNPs with functional relevance [34]. In this GWAS involving 1,644 non-Hispanic whites with asthma from six clinical trials, each subject was genotyped for 469,884 SNPs with reference to their associations with BDR. The top SNPs from primary GWAS were then replicated in two independent populations with 501 European Americans from Severe Asthma Research Program and 550 Dutch from Dutch Asthma GWAS respectively. They identified rs295137 near SPATS2L to have the strongest genetic association. Subjects with TT genotype of this SNP had a larger BDR than those with CC or TC genotypes. In the downstream experiments, the authors selected SPATS2L for functional validation by siRNA knockdown and Western blotting analyses. Knockdown model of this gene led to increased $\beta_{2}$-adrenoceptor levels, suggesting that SPATS2L may be an important regulator of $\beta_{2}$-adrenoceptor expression.

Another GWAS identified collagen type XXII a1 (COL22A1) gene as a novel locus for BDR among asthmatic patients [35]. In this study, 403 white trios from CAMP were recruited and each of them genotyped for 534,290 SNPs. A total of 1,397 SNPs were selected for subsequent genotyping on participants of three asthma trials. Thirteen SNPs with top genetic associations were then subjected to second phase replication. Through this multi-stage approach, they found significant association between BDR and an intergenic SNP (rs11252394) on chromosome 10. This SNP is located proximal to several excellent biological candidates for BDR. Despite it being not proximal to a gene within $50 \mathrm{~kb}$, this SNP is located on a genomic region where there are several excellent biological candidates within $2.5 \mathrm{Mb}$, including protein kinase C $\theta$ (PRKCQ), interleukin receptors (IL15RA and IL2RA) and Krüppel-like factor 6 (KLF6). All four genes have been previously reported to regulate BDR or pulmonary inflammation using in vitro cellular and murine models. More research work is needed to identify the causative variant in this genomic region. Another SNP (rs6988229) in the intron of COL22A1also showed strong replication signals in the initial GWAS population.

Although not a whole-genome study, Duan and coworkers reported important pharmacogenetic findings from a cellular drug-response study. They genotyped 1,116 SNPs of 98 transcription factor (TF) genes for association with BDR in asthma patients [36]. These TFs were shown in a cellular drug response study to be regulated by the $\beta_{2}$ agonist isoproterenol in a pro-asthmatic culture milieu. Four hundred and three non-Hispanic White asthmatic children and their parents were studied in the discovery set, and potentially significant SNPs were further genotyped in three adult asthma trials. One non-coding SNP (rs892940) located 5' of the thyroid hormone receptor- $\beta$ gene (THRB) was associated with BDR in the childhood population and two adult populations. Additional replication studies are needed to confirm the importance of this gene in modulating BDR.

\section{Leukotriene modifiers}

Leukotriene modifiers are positioned either as mono therapy for patients with mild persistent asthma, especially in young and nonatopic children with viral-associated wheeze, or as an add-on treatment 
for those with more severe and uncontrolled asthma [5]. This class of drugs inhibits the action of leukotrienes, which are a family of products synthesized from the metabolism of arachidonic acid that mediate important biological processes relevant to asthma (e.g. smooth muscle contraction, mucus secretion and allergic airway inflammation) [37]. There are two major classes of leukotriene modifiers that are used to treat asthma: LTRAs including montelukast, zafirlukast and pranlukast as well as 5-lipoxygenase (5-LO) inhibitor (zileuton). There is substantial inter-individual variability in response to leukotriene modifiers [11,38], which led to active pharmacogenetic research that tried to dissect the genetic basis for such heterogeneity in drug response. Thus far, there is no whole-genome study for leukotriene modifiers. Nonetheless, a number of pharmacogenetic studies were published on polymorphic markers that were relevant to the treatment response to leukotriene modifiers. Multiple gene loci have now been shown to have consistent association with treatment effects of these drugs. In an early pharmacogenetic study, Drazen et al. analyzed the 5-lipoxygenase gene (ALOX5) in asthmatics who received monotherapy with a 5-LO inhibitor ABT-761 [39]. Subjects with at least one wild-type allele experienced greater improvements in $\mathrm{FEV}_{1}$ than patients with any two of the minor alleles (mean values $19 \%$ versus $1 \%$ ). The importance of this gene was replicated in a smaller cohort of 61 asthma patients who received add-on montelukast treatment [40].

In addition to $A L O X 5$, pharmacogenetic studies of leukotriene modifiers were performed on other genes along the leukotriene pathway. Lima et al. genotyped 28 SNPs from five leukotriene pathway genes on 61 whites with poorly controlled, mild-to-moderate persistent asthma who were treated with single therapy of montelukast [38]. The SNPs of both ALOX5 and ABCC1 (ATP-binding cassette, subfamily C, member 1gene; rs119774) were significantly associated with $\mathrm{FEV}_{1}$ change in response to this LTRA. Besides, a SNP (rs730012) located on promoter of leukotriene $\mathrm{C}_{4}$ synthase (LTC4S) was also found to be associated with a reduced risk of asthma exacerbation while an intronic SNP (rs2660845) in leukotriene $\mathrm{A}_{4}$ hydrolase (LTA4H) was associated with increased exacerbation rate of asthma. These studies identified leukotriene pathway-related genes as determinants for the variability in drug response to leukotriene modifiers. To provide a broader perspective, larger studies desirably through genome-wide approach are needed to identify novel gene targets for leukotriene modifiers.

\section{Future Research Directions}

A number of Pharmacogenomic approaches including GWAS, DNA microarray for gene expression, next-generation sequencing (NGS), and systems biologyand genome-wide epigenetic testing are available for research to delineate therapeutic responses to antiasthma medications. From the above discussion, GWAS and wholegenome microarray shave already found their applications in asthma pharmacogenomic studies by identifying several novel candidate genes that modulated treatment responses to asthma drugs (Table 1). However, there are several limitations for such approaches. Firstly, most whole-genome studies are without apriori hypothesis. Any positive finding does not imply that the identified gene indeed exerts direct and mechanistic effects on pharmacokinetic or pharmacodynamic handling of anti-asthma medications. Without downstream validation works, these pharmacogenomic studies only imply the presence of genetic susceptibility factors on certain parts of the human genome. Secondly, it is difficult to account for possible interactions among the candidate genes for treatment responses in view of the complex computational and statistical reasons.
Systems biology, high-throughput NGS and genome-wide testing for epigenetic effects are emerging investigative approaches for pharmacogenomic research. Systems biology is a relatively new approach to the study and modeling of biological systems [41]. It explores the entirety of a biological system, including the network of interactions between different factors and components in the system. Therefore, systems biology can help researchers to capture complex interactions between genetic factors and environmental exposures so as to develop an improved understanding on the responses to anti-asthma drugs. Owing to the complex nature of asthma, pharmacogenomics phenotypes are likely to be determined by a combination of common and rare variants that predict drug responses. Considering that, NGS can provide fruitful answers to asthma pharmacogenomic research due to its ability to discover rare variations that are not identified through tagging strategies in GWAS [42,43]. For instance, our group has recently completed NGS of 10 asthma loci that were reported by a large Consortium-based GWAS [44] using Roche 454 pyrosequencing [45]. Analysis of 224 SNPs that were common to our subjects and different populations under 1000 Genomes Project revealed that these SNPs were grouped into 16 mostly small haplotype blocks in Hong Kong children, which was strikingly different from the smaller numbers of larger haplotype blocks found in other Asian, central European, Puerto Ricans and African populations (submitted for publication). Thus, NGS will generate finer, more accurate and ethnic-specific nucleotide sequences to facilitate the selection of tagging SNPs for genomic studies. Despite these technological advances, it is unlikely that any one approach will be sufficient to identify all genetic determinants for therapeutic responses to anti-asthma drugs. All the above investigative approaches are believed to have a place in the future of pharmacogenomic research for asthma.

\section{Conclusions}

During the past several years, significant progress in pharmacogenomics research has been made that identified increasing numbers of genetic factors that contribute to the heterogeneity in the treatment responses to different anti-asthma drugs. Whole-genome studies identify CLCA1, periostin, serpinB2, FKBP51, NFKB, GLCCI1 and $\mathrm{T}$ gene to be genetic determinants for ICS response. For the bronchodilator responses, ARG1, CRHR2, SPATS2L and COL22A1 are likely to be the important gene targets. Nonetheless, any novel gene target thus discovered will need to be supported by downstream in vitro and in vivo researches as well as validation works in independent populations of varying ethnicity. Adequate bioinformatics support is also essential in view of the enormous amount of whole-genome data involved. These findings will ultimately allow us to choose among asthma therapies that will likely be effective to any particular patient. In an attempt to achieve the goal of personalizing asthma pharmacotherapy, more resources and collaborative efforts are required to be spent on the domain of pharmacogenomics research.

\section{Acknowledgements}

This review and our genotyping works were supported by Research Grants Council General Research Fund (469908 and 470909) of Hong Kong SAR Government and Research Committee Group Research Scheme (3110034, 3110060 and 3110087 ) of Chinese University of Hong Kong.

\section{References}

1. Masoli M, Fabian D, Holt S, Beasley R (2004) The global burden of asthma: executive summary of the GINA Dissemination Committee report. Allergy 59 $469-478$.

2. Newman-Taylor A (1995) Environmental determinants of asthma. Lancet 345 : 296-299. 
3. Wiesch DG, Meyers DA, Bleecker ER (1999) Genetics of asthma. J Allergy Clinlmmunol 104: 895-901.

4. Ober C (2005) Perspectives on the past decade of asthma genetics. J Allergy Clinlmmunol 116: 274-278.

5. Global Initiative for Asthma (2012). Global strategy for asthma management and prevention.

6. British Thoracic Society and Scottish Intercollegiate Guidelines Network (2012) British guideline on the management of asthma: A national clinical guideline 37-51.

7. Drazen JM, Silverman EK, Lee TH (2000) Heterogeneity of therapeutic responses in asthma. Br Med Bull 56: 1054-1070.

8. Lemanske RF Jr, Mauger DT, Sorkness CA, Jackson DJ, Boehmer SJ, et al (2010) Step-up therapy for children with uncontrolled asthma receiving inhaled corticosteroids. N Engl J Med 362: 975-985.

9. Abecasis GR, Auton A, Brooks LD, DePristo MA, et al. (2012) An integrated map of genetic variation from 1,092 human genomes. Nature 491: 56-65.

10. National Asthma Education and Prevention Program (2007) Expert Pane Report 3 (EPR-3): Guidelines for the Diagnosis and Management of AsthmaSummary Report 2007. J Allergy Clinlmmunol 120: S94-S138.

11. Malmstrom K, Rodriguez-Gomez G, Guerra J, Villaran C, Piñeiro A, et al. (1999) Oral montelukast, inhaled beclomethasone, and placebo for chronic asthma. A randomized, controlled trial. Montelukast/Beclomethasone Study Group. Ann Intern Med 130: 487-495.

12. Szefler SJ, Phillips BR, Martinez FD, Chinchilli VM, Lemanske RF, et al. (2005) Characterization of within-subject responses to fluticasone and montelukast in childhood asthma. J Allergy ClinImmunol 115: 233-242.

13. Woodruff PG, Boushey HA, Dolganov GM, Barker CS, Yang YH, et al. (2007) Genome-wide profiling identifies epithelial cell genes associated with asthma and with treatment response to corticosteroids. ProcNatIAcadSci U S A 104: 15858-15863.

14. Hakonarson H, Bjornsdottir US, Halapi E, Bradfield J, Zink F, et al. (2005) Profiling of genes expressed in peripheral blood mononuclear cells predicts glucocorticoid sensitivity in asthma patients. ProcNatlAcadSci U S A 102: 14789-14794.

15. Tantisira KG, Lasky-Su J, Harada M, Murphy A, Litonjua AA, et al. (2011) Genomewide association between GLCCl1 and response to glucocorticoid therapy in asthma. N Engl J Med 365: 1173-1183.

16. McGeachie MJ, Wu AC, Chang HH, Lima JJ, Peters SP, et al. (2012) Predicting inhaled corticosteroid response in asthma with two associated SNPs. Pharmacogenomics $\mathrm{J}$

17. Tantisira KG, Damask A, Szefler SJ, Schuemann B, Markezich A, et al (2012) Genome-wide association identifies the $T$ gene as a novel asthma pharmacogenetic locus. Am J RespirCrit Care Med 185: 1286-1291.

18. Sears MR, Lötvall J (2005) Past, present and future--beta2-adrenoceptor agonists in asthma management. Respir Med 99: 152-170.

19. Salpeter SR, Buckley NS, Ormiston TM, Salpeter EE (2006) Meta-analysis: effect of long-acting beta-agonists on severe asthma exacerbations and asthma-related deaths. Ann Intern Med 144: 904-912.

20. Lazarus SC, Boushey HA, Fahy JV, Chinchilli VM, Lemanske RF Jr, et al. (2001) Long-acting beta2-agonist monotherapyvs continued therapy with inhaled corticosteroids in patients with persistent asthma: a randomized controlled trial. JAMA 285: 2583-2593.

21. Johnson M (2006) Molecular mechanisms of beta(2)-adrenergic receptor function, response, and regulation. J Allergy Clinlmmunol 117: 18-24.

22. Thakkinstian A, McEvoy M, Minelli C, Gibson P, Hancox B, et al. (2005) Systematic review and meta-analysis of the association between \{beta\}2adrenoceptor polymorphisms and asthma: a HuGE review. Am J Epidemiol 162: 201-211.

23. Taylor DR, Epton MJ, Kennedy MA, Smith AD, Iles S, et al. (2005) Bronchodilator response in relation to beta2-adrenoceptor haplotype in patients with asthma. Am J RespirCrit Care Med 172: 700-703.

24. Bleecker ER, Postma DS, Lawrance RM, Meyers DA, Ambrose HJ, et al. (2007) Effect of ADRB2 polymorphisms on response to longacting beta2-agonist therapy: a pharmacogenetic analysis of two randomised studies. Lancet 370: 2118-2125.
25. Wechsler ME, Kunselman SJ, Chinchilli VM, Bleecker E, Boushey HA, et al. (2009) Effect of beta2-adrenergic receptor polymorphism on response to longacting beta2 agonist in asthma (LARGE trial): a genotype-stratified, randomised, placebo-controlled, crossover trial. Lancet 374: 1754-1764.

26. Bleecker ER, Meyers DA, Bailey WC, Sims AM, Bujac SR, et al. (2012) ADRB2 polymorphisms and budesonide/formoterol responses in COPD. Chest 142 320-328.

27. Litonjua AA, Lasky-Su J, Schneiter K, Tantisira KG, Lazarus R, et al. (2008) ARG1 is a novel bronchodilator response gene: screening and replication in four asthma cohorts. Am J RespirCrit Care Med 178: 688-694.

28. Zimmermann N, King NE, Laporte J, Yang M, Mishra A, et al. (2003) Dissection of experimental asthma with DNA microarray analysis identifies arginase in asthma pathogenesis. J Clin Invest 111: 1863-1874.

29. Mabalirajan U, Ahmad T, Leishangthem GD, Joseph DA, Dinda AK, et al. (2010) Beneficial effects of high dose of L-arginine on airway hyperresponsiveness and airway inflammation in a murine model of asthma. J Allergy Clinlmmuno 125: 626-635.

30. North ML, Khanna N, Marsden PA, Grasemann H, Scott JA (2009) Functionally important role for arginase 1 in the airway hyperresponsiveness of asthma. Am J Physiol Lung Cell MolPhysiol 296: L911-920.

31. Breton CV, Byun HM, Wang X, Salam MT, Siegmund K, et al. (2011) DNA methylation in the arginase-nitric oxide synthase pathway is associated with exhaled nitric oxide in children with asthma. Am J RespirCrit Care Med 184 191-197.

32. Sy HY, Ko FW, Chu HY, Chan IH, Wong GW, et al. (2012) Asthma and bronchodilator responsiveness are associated with polymorphic markers of ARG1, CRHR2 and chromosome 17q21. Pharmacogenet Genomics 22: 517524.

33. Moffatt MF, Kabesch M, Liang L, Dixon AL, Strachan D, et al. (2007) Genetic variants regulating ORMDL3 expression contribute to the risk of childhood asthma. Nature 448: 470-473.

34. Himes BE, Jiang X, Hu R, Wu AC, Lasky-Su JA, et al. (2012) Genomewide association analysis in asthma subjects identifies SPATS2L as a novel bronchodilator response gene. PLoS Genet 8: e1002824.

35. Duan QL, Lasky-Su J, Himes BE, Qiu W, Litonjua AA, et al. (2013) A genome-wide association study of bronchodilator response in asthmatics. Pharmacogenomics $\mathrm{J}$.

36. Duan QL, Du R, Lasky-Su J, Klanderman BJ, Partch AB, et al. (2013) A polymorphism in the thyroid hormone receptor gene is associated with bronchodilator response in asthmatics. Pharmacogenomics J 13: 130-136.

37. Peters-Golden M, Henderson WR Jr (2007) Leukotrienes. N Engl J Med 357: 1841-1854.

38. Lima JJ (2007) Treatment heterogeneity in asthma: genetics of response to leukotriene modifiers. MolDiagnTher 11: 97-104.

39. Drazen JM, Yandava CN, Dubé L, Szczerback N, Hippensteel R, et al. (1999) Pharmacogenetic association between ALOX5 promoter genotype and the response to anti-asthma treatment. Nat Genet 22: 168-170.

40. Telleria JJ, Blanco-Quiros A, Varillas D, Armentia A, Fernandez-Carvajal I, et al. (2008) ALOX5 promoter genotype and response to montelukast in moderate persistent asthma. Respir Med 102: 857-861.

41. Dahlin A, Tantisira KG (2012) Integrative systems biology approaches in asthma pharmacogenomics. Pharmacogenomics 13: 1387-1404.

42. Cookson WO, Moffatt MF (2011) Genetics of complex airway disease. Proc Am ThoracSoc 8: 149-153.

43. Roukos DH, Ziogas DE, Baltogiannis GG, Katsouras CS, Bechlioulis A, et al. (2013) Novel Next-Generation Sequencing and Networks-Based Therapeutic Targets: Realistic more Effective Drug Design and Discovery. Curr Pharm Des

44. Moffatt MF, Gut IG, Demenais F, Strachan DP, Bouzigon E, et al. (2010) A largescale, consortium-based genomewide association study of asthma. $\mathrm{N}$ Engl J Med 363: 1211-1221.

45. Leung TF, Law P, Tse LY, Sy HY, Tsui SK (2012) Characterization of genetic epidemiology for GWAS-identified asthma susceptibility loci in Chinese by next generation sequencing. J Allergy ClinImmunol 129: AB137. 